\title{
Coherent and convex monetary risk measures for unbounded càdlàg processes
}

\author{
Patrick Cheridito • Freddy Delbaen \\ Michael Kupper
}

Published online: 11 August 2006

(C) Springer-Verlag 2006

\begin{abstract}
Assume that the random future evolution of values is modelled in continuous time. Then, a risk measure can be viewed as a functional on a space of continuous-time stochastic processes. In this paper we study coherent and convex monetary risk measures on the space of all càdlàg processes that are adapted to a given filtration. We show that if such risk measures are required to be real-valued, then they can only depend on a stochastic process in a way that is uninteresting for many applications. Therefore, we allow them to take values in $(-\infty, \infty]$. The economic interpretation of a value of $\infty$ is that the corresponding financial position is so risky that no additional amount of money can make it acceptable. The main result of the paper gives different characterizations of coherent or convex monetary risk measures on the space of all bounded adapted càdlàg processes that can be extended to coherent or convex monetary
\end{abstract}

P. Cheridito and M. Kupper were supported by the Swiss Science Agency.

F. Delbaen and M. Kupper were supported by Credit Suisse and NCCR-FINRISK.

Due to errors during the typesetting process, this article was published incorrectly in Finance Stoch 9(3):369-387 (2005). The address of the first author was printed incorrectly, and in the whole paper the angular brackets \langle\rangle were misprinted as [ ]. The complete corrected article is given here. The online version of the original paper can be found at: http://dx.doi.org/10.1007/s00780-004-0150-7.

P. Cheridito $(\bowtie)$

ORFE, Princeton University, Princeton, NJ 08544, USA

e-mail: dito@princeton.edu

F. Delbaen · M. Kupper

Departement für Mathematik, ETH Zürich, 8092 Zürich, Switzerland

e-mail: delbaen@math.ethz.ch

M. Kupper

e-mail: kupper@math.ethz.ch 
risk measures on the space of all adapted càdlàg processes. As examples we discuss a new approach to measure the risk of an insurance company and a coherent risk measure for unbounded càdlàg processes induced by a so called m-stable set.

Keywords Coherent risk measures - Convex monetary risk measures . Coherent utility functionals - Concave monetary utility functionals - Unbounded càdlàg processes · Extension of risk measures

Mathematics Subject Classification (2000) 91B30 - 91B16 - 60G07 · 52A07 . 46A55 - 46A20

JEL Classification D81 - C60 - G18

\section{Introduction}

The notion of a coherent risk measure was introduced in Artzner et al. $[1,2]$ and has been extended to more general setups by Delbaen [7-9], Artzner et al. [3], Riedel [16] and Cheridito et al [5]. The more general concept of a convex monetary risk measures was established in Föllmer and Schied [12-14] and Frittelli and Rosazza Gianin [15]. In [5], the future evolution of discounted values is modelled with essentially bounded càdlàg processes, and a risk measure is a real-valued functional on the space $\mathcal{R}^{\infty}$ of equivalence classes of adapted, essentially bounded, càdlàg processes on a filtered probability space $\left(\Omega, \mathcal{F},\left(\mathcal{F}_{t}\right)_{t \in[0, T]}, P\right)$ satisfying the usual assumptions. It is shown in [5] that every convex monetary risk measure on $\mathcal{R}^{\infty}$ that is so called continuous for bounded decreasing sequences can be represented in terms of sigma-additive linear functionals. Such a representation can be useful for computational purposes, the verification of properties or the transformation of risk measures. The space $\mathcal{R}^{\infty}$ easily lends itself to the application of duality theory and is therefore well suited for a functional analytic treatment of risk measures that depend on continuous-time stochastic processes. On the other hand, most examples of stochastic processes playing a role in financial models are unbounded. In this paper, we study coherent and convex monetary risk measures on the space $\mathcal{R}^{0}$ of equivalence classes of all adapted càdlàg processes on $\left(\Omega, \mathcal{F},\left(\mathcal{F}_{t}\right)_{t \in[0, T]}, P\right)$. Our approach is to view them as extensions of risk measures on $\mathcal{R}^{\infty}$, and the main result of this paper is a characterization of coherent and convex monetary risk measures on $\mathcal{R}^{\infty}$ that can be extended to coherent or convex monetary risk measure on $\mathcal{R}^{0}$. Consistent with the interpretation that a monetary risk measure yields the amount of money that has to be added to a financial position to make it acceptable (see, for instance Atzner et al. [2], Delbaen [7] or Föllmer and Schied [14]), such a risk measure on $\mathcal{R}^{\infty}$ is real-valued. On the other hand, we show that if one requires coherent and convex monetary risk measures on $\mathcal{R}^{0}$ to be real-valued, one is excluding many interesting examples. Therefore, we allow them to take values in $(-\infty, \infty]$. A value of $\infty$ then means that the 
corresponding discounted value process is so risky that no amount of additional money can make it acceptable.

Instead of starting with real-valued coherent and convex monetary risk measures on $\mathcal{R}^{\infty}$ and then extending them to $\mathcal{R}^{0}$, one could right away consider risk measures on one of the spaces $\mathcal{R}^{p}, p \in[1, \infty)$, of equivalence classes of adapted, càdlàg processes $\left(X_{t}\right)_{t \in[0, T]}$ such that $\mathrm{E}\left[\left(\sup _{t \in[0, T]}\left|X_{t}\right|\right)^{p}\right]<\infty$. For instance, it is shown in Proposition 3.8 of Cheridito et al. [5] that every real-valued convex monetary risk measure on $\mathcal{R}^{p}$ is upper semicontinuous in the norm-topology of $\mathcal{R}^{p}$ and can therefore be represented in terms of sigma-additive linear functionals. However, this approach has several drawbacks. First of all, if the probability space is not finite, then all the spaces $\mathcal{R}^{p}, p \in[1, \infty)$, are strictly smaller than $\mathcal{R}^{0}$ and cannot accommodate non-integrable processes that could be interesting for financial modelling. On the other hand, since $\mathcal{R}^{\infty}$ is the smallest of all the spaces $\mathcal{R}^{p}, p \in[1, \infty]$, the set of real-valued convex monetary risk measures on $\mathcal{R}^{p}$ is largest for $p=\infty$. Hence, by focusing on real-valued convex monetary risk measures on one of the spaces $\mathcal{R}^{p}, p \in[1, \infty)$, one might miss interesting examples of convex monetary risk measures that are real-valued on $\mathcal{R}^{\infty}$ but can also take the value $\infty$ when extended to $\mathcal{R}^{0}$. At last, in many situations in risk management it is not clear what probability to assign to certain future events and a whole set of different probability measures is taken into consideration. The spaces $\mathcal{R}^{\infty}$ and $\mathcal{R}^{0}$ are invariant if $P$ is changed to another probability measure as long as it is equivalent to $P$. This is not the case for the spaces $\mathcal{R}^{p}$, $p \in[1, \infty)$.

We emphasize that while we model the evolution of discounted future values over a whole time interval, the risk measures considered in this paper are, like in Cheridito et al. [5], static, as we calculate the risk of a value process only at the beginning of the time interval. Of course, it is also important to study how risk measures should be updated in a consistent way as more information is becoming available over time. This question is for instance, addressed in Artzner et al. [3], Delbaen [9], Wang [19], Roorda et al. [18], Riedel [16] and Cheridito et al. [6].

The structure of the paper is as follows: In section 2, we recall the most important definitions and results from Cheridito et al. [5] concerning coherent and convex monetary risk measures on $\mathcal{R}^{\infty}$. In section 3 , we prove a theorem which shows that the requirement that coherent or convex monetary risk measures be real-valued is too much of a restriction and that it is better to let them take values in $(-\infty, \infty]$. This leads to our definition of coherent and convex monetary risk measures on $\mathcal{R}^{0}$. Then, we state the paper's main result, which gives a characterization of coherent and convex monetary risk measures on $\mathcal{R}^{\infty}$ that can be extended to coherent or convex monetary risk measures on $\mathcal{R}^{0}$. Section 4 contains the proof of the main result. In section 5 , we give two examples of a coherent risk measure on $\mathcal{R}^{0}$. The first one is related to the Cramér-Lundberg approach to measuring the risk of an insurance company. The second one is motivated by results in Delbaen [9], where time consistency properties of dynamic risk measures that depend on one-dimensional random variables are studied. 


\section{Preliminaries}

Let $T \in[0, \infty)$ and $\left(\Omega, \mathcal{F},\left(\mathcal{F}_{t}\right)_{t \in[0, T]}, P\right)$ be a filtered probability space that satisfies the usual assumptions, that is, the probability space $(\Omega, \mathcal{F}, P)$ is complete, the filtration $\left(\mathcal{F}_{t}\right)$ is right-continuous and $\mathcal{F}_{0}$ contains all null-sets of $\mathcal{F}$. We identify indistinguishable stochastic processes, and statements involving random variables or stochastic processes are understood in the almost sure sense. For instance, by a càdlàg process we mean a stochastic process whose paths almost surely are right-continuous and have left limits, and for stochastic processes $\left(X_{t}\right)_{t \in[0, T]}$ and $\left(Y_{t}\right)_{t \in[0, T]}, X \geq Y$ means that for almost all $\omega$, $X_{t}(\omega) \geq Y_{t}(\omega)$ for all $t \in[0, T]$. For $1 \leq p \leq \infty$, let

$$
\mathcal{R}^{p}:=\left\{\begin{array}{l|l}
X:[0, T] \times \Omega \rightarrow \mathbb{R} & \begin{array}{l}
X \text { càdlàg, }\left(\mathcal{F}_{t}\right) \text {-adapted } \\
\|X\|_{\mathcal{R}^{p}}<\infty
\end{array}
\end{array}\right\},
$$

where $\|X\|_{\mathcal{R}^{p}}:=\left\|X^{*}\right\|_{p}$ and $X^{*}:=\sup _{0 \leq t \leq T}\left|X_{t}\right|$. It is easy to see that $\|\cdot\|_{p}$ is a norm on $\mathcal{R}^{p}$, and $\mathcal{R}^{p}$ equipped with this norm is a Banach space. For a stochastic process $b:[0, T] \times \Omega \rightarrow \mathbb{R}$ with right-continuous paths of finite variation, we denote by $b=b^{+}-b^{-}$the minimal decomposition of $b$ into non-negative increasing right-continuous processes $b^{+}$and $b^{-}$. The variation of such a process is given by the random variable $\operatorname{Var}(b):=b^{+}(T)+b^{-}(T)$. If $b$ is optional (predictable), both processes $b^{+}$and $b^{-}$are optional (predictable). For $q \in[1, \infty]$, we set

$$
\mathcal{A}^{q}:=\left\{\begin{array}{l|l}
a:[0, T] \times \Omega \rightarrow \mathbb{R}^{2} & \begin{array}{l}
a=\left(a^{\mathrm{pr}}, a^{\mathrm{op}}\right) \\
a^{\mathrm{pr}}, a^{\mathrm{op}} \text { right continuous, finite variation } \\
a^{\mathrm{pr}} \text { predictable, } a_{0}^{\mathrm{pr}}=0 \\
a^{\mathrm{op}} \text { optional, purely discontinuous } \\
\operatorname{Var}\left(a^{\mathrm{pr}}\right)+\operatorname{Var}\left(a^{\mathrm{op}}\right) \in L^{q}
\end{array}
\end{array}\right\},
$$

It can be shown that $\mathcal{A}^{q}$ with the norm

$$
\|a\|_{\mathcal{A}^{q}}:=\left\|\operatorname{Var}\left(a^{\mathrm{pr}}\right)+\operatorname{Var}\left(a^{\mathrm{op}}\right)\right\|_{q}, \quad a \in \mathcal{A}^{q},
$$

is also a Banach space. We set

$$
\mathcal{A}_{+}^{q}:=\left\{a=\left(a^{\mathrm{pr}}, a^{\mathrm{op}}\right) \in \mathcal{A}^{q} \mid a^{\mathrm{pr}} \text { and } a^{\mathrm{op}} \text { are non-negative and increasing }\right\}
$$

and

$$
\mathcal{D}_{\sigma}:=\left\{a \in \mathcal{A}_{+}^{1} \mid\|a\|_{\mathcal{A}^{1}}=1\right\} .
$$


It can easily be checked that for all $p, q \in[1, \infty]$ such that $p^{-1}+q^{-1}=1$,

$$
\langle X, a\rangle:=\mathrm{E}\left[\int_{[0, T]} X_{t-} \mathrm{d} a_{t}^{\mathrm{pr}}+\int_{[0, T]} X_{t} \mathrm{~d} a_{t}^{\mathrm{op}}\right]
$$

is a well-defined bilinear form on $\mathcal{R}^{p} \times \mathcal{A}^{q}$, and

$$
|\langle X, a\rangle| \leq\|X\|_{\mathcal{R}^{p}}\|a\|_{\mathcal{A}} q, \quad \text { for all } X \in \mathcal{R}^{p} \text { and } a \in \mathcal{A}^{q}
$$

Note that for $T=0, \mathcal{R}^{p}=L^{p}\left(\Omega, \mathcal{F}_{0}, P\right)$ and $\mathcal{A}^{q}=L^{q}\left(\Omega, \mathcal{F}_{0}, P\right)$. Therefore, our framework includes the one time-step setup, where a risk measure is a functional on a space of random variables. The space

$$
\mathcal{R}^{0}:=\left\{X:[0, T] \times \Omega \rightarrow \mathbb{R} \mid X \text { càdlàg, }\left(\mathcal{F}_{t}\right) \text {-adapted }\right\} \text {. }
$$

with the metric

$$
d(X, Y):=\mathrm{E}\left[(X-Y)^{*} \wedge 1\right]
$$

is complete but not locally convex.

We call any mapping $\rho: \mathcal{R}^{\infty} \rightarrow \mathbb{R}$ a risk measure on $\mathcal{R}^{\infty}$. We find it more convenient to work with the negative of a risk measure $\phi=-\rho$. We call $\phi$ the utility functional corresponding to the risk measure $\rho$.

Definition 2.1 A concave monetary utility functional on $\mathcal{R}^{\infty}$ is a mapping $\phi$ : $\mathcal{R}^{\infty} \rightarrow \mathbb{R}$ with the following properties:

(1) $\phi(\lambda X+(1-\lambda) Y) \geq \lambda \phi(X)+(1-\lambda) \phi(Y)$, for all $X, Y \in \mathcal{R}^{\infty}$ and $\lambda \in[0,1]$

(2) $\phi(X) \leq \phi(Y)$, for all $X, Y \in \mathcal{R}^{\infty}$ with $X \leq Y$

(3) $\phi(X+m)=\phi(X)+m$, for all $X \in \mathcal{R}^{\infty}$ and $m \in \mathbb{R}$.

We call a concave utility functional $\phi$ on $\mathcal{R}^{\infty}$ a coherent utility functional on $\mathcal{R}^{\infty}$ if it has the additional property:

(4) $\phi(\lambda X)=\lambda \phi(X)$, for all $X \in \mathcal{R}^{\infty}$ and $\lambda \in \mathbb{R}_{+}$.

We call the negative $\rho=-\phi$ of a concave monetary utility functional $\phi$ on $\mathcal{R}^{\infty}$, a convex monetary risk measure on $\mathcal{R}^{\infty}$. If $\phi$ is a coherent utility functional on $\mathcal{R}^{\infty}$, we call $\rho$ a coherent risk measure on $\mathcal{R}^{\infty}$.

The acceptance set $\mathcal{C}$ corresponding to a concave monetary utility functional $\phi$ on $\mathcal{R}^{\infty}$ is given by

$$
\mathcal{C}:=\left\{X \in \mathcal{R}^{\infty} \mid \phi(X) \geq 0\right\}
$$

It is convex and has the following property: If $X, Y \in \mathcal{R}^{\infty}, X \in \mathcal{C}$ and $X \leq Y$, then $Y \in \mathcal{C}$ as well. If $\phi$ is coherent, then $\mathcal{C}$ is a convex cone. 
For a concave monetary utility functional $\phi$ on $\mathcal{R}^{\infty}$, we define its conjugate by

$$
\phi^{*}(a):=\inf _{X \in \mathcal{R}^{\infty}}\{\langle X, a\rangle-\phi(X)\}, \quad a \in \mathcal{A}^{1}
$$

It can be shown (see Föllmer and Schied [12]) that for all $a \in \mathcal{D}_{\sigma}$,

$$
\phi^{*}(a)=\inf _{X \in \mathcal{C}}\langle X, a\rangle
$$

We call a function $\gamma: \mathcal{D}_{\sigma} \rightarrow[-\infty, \infty)$ a penalty function if

$$
-\infty<\sup _{a \in \mathcal{D}_{\sigma}} \gamma(a)<\infty
$$

Definition 2.2 We say that a concave monetary utility functional $\phi$ on $\mathcal{R}^{\infty}$ satisfies the Fatou property if

$$
\limsup _{n \rightarrow \infty} \phi\left(X^{n}\right) \leq \phi(X)
$$

for every bounded sequence $\left(X^{n}\right)_{n \geq 1} \subset \mathcal{R}^{\infty}$ and $X \in \mathcal{R}^{\infty}$ such that $\left(X^{n}-X\right)^{*} \stackrel{P}{\rightarrow} 0$.

We say that $\phi$ is continuous for bounded decreasing sequences if

$$
\lim _{n \rightarrow \infty} \phi\left(X^{n}\right)=\phi(X)
$$

for every decreasing sequence $\left(X^{n}\right)_{n \geq 1} \subset \mathcal{R}^{\infty}$ such that $\left(X^{n}-X\right)^{*} \stackrel{P}{\rightarrow} 0$ for some $X \in \mathcal{R}^{\infty}$.

The following theorem and corollary are proved in Cheridito et al. [5].

Theorem 2.3 The following are equivalent:

(1) $\phi$ is a mapping defined on $\mathcal{R}^{\infty}$ that can be represented as

$$
\phi(X)=\inf _{a \in \mathcal{D}_{\sigma}}\{\langle X, a\rangle-\gamma(a)\}, \quad X \in \mathcal{R}^{\infty},
$$

for a penalty function $\gamma: \mathcal{D}_{\sigma} \rightarrow[-\infty, \infty)$.

(2) $\phi$ is a concave monetary utility functional on $\mathcal{R}^{\infty}$ whose acceptance set $\mathcal{C}:=\left\{X \in \mathcal{R}^{\infty} \mid \phi(X) \geq 0\right\}$ is $\sigma\left(\mathcal{R}^{\infty}, \mathcal{A}^{1}\right)$-closed

(3) $\phi$ is a concave monetary utility functional on $\mathcal{R}^{\infty}$ that satisfies the Fatou property.

(4) $\phi$ is a concave monetary utility functional on $\mathcal{R}^{\infty}$ that is continuous for bounded decreasing sequences. 
Moreover, if (1)-(4) are satisfied, then the restriction of $\phi^{*}$ to $\mathcal{D}_{\sigma}$ is a penalty function, $\phi^{*}(a) \geq \gamma\left(\right.$ a) for all $a \in \mathcal{D}_{\sigma}$, and the representation (2.3) also holds if $\gamma$ is replaced by $\phi^{*}$.

Corollary 2.4 The following are equivalent:

(1) $\phi$ is a mapping defined on $\mathcal{R}^{\infty}$ that can be represented as

$$
\phi(X)=\inf _{a \in \mathcal{Q}_{\sigma}}\langle X, a\rangle, \quad X \in \mathcal{R}^{\infty},
$$

for a non-empty set $\mathcal{Q}_{\sigma} \subset \mathcal{D}_{\sigma}$.

(2) $\phi$ is a coherent utility functional on $\mathcal{R}^{\infty}$ whose acceptance set

$\mathcal{C}:=\left\{X \in \mathcal{R}^{\infty} \mid \phi(X) \geq 0\right\}$ is $\sigma\left(\mathcal{R}^{\infty}, \mathcal{A}^{1}\right)$-closed.

(3) $\phi$ is a coherent utility functional on $\mathcal{R}^{\infty}$ that satisfies the Fatou property.

(4) $\phi$ is a coherent utility functional on $\mathcal{R}^{\infty}$ that is continuous for bounded decreasing sequences.

\section{Extension of risk measures from $\mathcal{R}^{\infty}$ to $\mathcal{R}^{0}$}

In this section we first extend Theorem 5.1 of Delbaen [7] and show that realvalued concave monetary utility functionals on $\mathcal{R}^{0}$ are of a very restricted form. Therefore, we allow them to take values in $[-\infty, \infty)$. Then, we we give conditions for concave monetary utility functionals on $\mathcal{R}^{\infty}$ to be extendable to concave monetary utility functionals on $\mathcal{R}^{0}$.

Assume that the probability space $\left(\Omega, \mathcal{F}_{0}, P\right)$ is the union of finitely many atoms and $\mathcal{P}_{\sigma}$ is a set of probability measures on $\left(\Omega, \mathcal{F}_{0}\right)$ that are absolutely continuous with respect to $P$. Then the map

$$
X \mapsto \inf _{Q \in \mathcal{P}_{\sigma}} \mathrm{E}_{Q}\left[X_{0}\right]
$$

is a real-valued coherent utility functional on $\mathcal{R}^{0}$. Hence, there are settings where real-valued coherent and concave monetary utility functionals on $\mathcal{R}^{0}$ exist. However, the next theorem shows that to have interesting coherent and concave monetary utility functionals on $\mathcal{R}^{0}$, one should not require them to be real-valued.

Theorem 3.1 Let $f: \mathcal{R}^{0} \rightarrow \mathbb{R}$ be a real-valued, increasing, concave function, $S$ a $[0, T]$-valued stopping time and $B \in \mathcal{F}_{S}$ such that $\left(B, \mathcal{F}_{S}^{B}, P\right)$ is atomless, where $\mathcal{F}_{S}^{B}:=\left\{A \cap B \mid A \in \mathcal{F}_{S}\right\}$. Then,

$$
f\left(X+Y \mathbf{1}_{B} \mathbf{1}_{[S, T]}\right)=f(X) \quad \text { for all } X, Y \in \mathcal{R}^{0} .
$$

Proof Assume that (3.1) does not hold. Then there exist $X^{0} \in \mathcal{R}^{0}$ and $Y^{1}, Y^{2}$ in the subspace

$$
\mathcal{R}_{S, B}^{0}:=\left\{X \mathbf{1}_{B} \mathbf{1}_{[S, T]} \mid X \in \mathcal{R}^{0}\right\}
$$


such that $\tilde{f}\left(Y^{1}\right)<\tilde{f}\left(Y^{2}\right)$, where the function $\tilde{f}: \mathcal{R}_{S, B}^{0} \rightarrow \mathbb{R}$ is given by

$$
\tilde{f}(Y):=f\left(X^{0}+Y\right)-f\left(X^{0}\right), \quad Y \in \mathcal{R}_{S, B}^{0} .
$$

Observe that $\tilde{f}$ is increasing, concave and $\tilde{f}(0)=0$. For every $n \in \mathbb{N}$, let $C_{n}$ be the convex set given by

$$
C_{n}:=\left\{Y \in \mathcal{R}_{S, B}^{0} \mid \tilde{f}(Y) \geq-n\right\} .
$$

Since $f$ is real-valued, $\bigcup_{n \geq 1} C_{n}=\mathcal{R}_{S, B}^{0}$, and therefore also, $\bigcup_{n \geq 1} \bar{C}_{n}=\mathcal{R}_{S, B}^{0}$, where $\bar{C}_{n}$ denotes the closure of $C_{n}$ in the metric (2.2). Hence, it follows from Baire's Theorem that there exists an $n_{0}$ such that $\bar{C}_{n_{0}}$ has non-empty interior, that is, there exists an $Y^{0} \in \mathcal{R}_{S, B}^{0}$ and an $\varepsilon>0$ such that

$$
\left\{Y \in \mathcal{R}_{S, B}^{0} \mid \mathrm{E}\left[\left(Y-Y^{0}\right)^{*} \wedge 1\right] \leq \varepsilon\right\} \subset \bar{C}_{n_{0}}
$$

By concavity, for all $j \geq 1$,

$$
\tilde{f}\left((j+1) Y^{1}-j Y^{2}\right) \leq(j+1) \tilde{f}\left(Y^{1}\right)-\tilde{j}\left(Y^{2}\right)=\tilde{f}\left(Y^{1}\right)-j\left[\tilde{f}\left(Y^{2}\right)-\tilde{f}\left(Y^{1}\right)\right],
$$

which shows that there exists a $Z^{0} \in \mathcal{R}_{S, B}^{0}$ such that $\tilde{f}\left(Z^{0}\right) \leq-\frac{1}{2}\left(n_{0}+1\right)$. Since $\left(B, \mathcal{F}_{S}^{B}, P\right)$ is atomless, there exist finitely many disjoint sets $B_{1}, \ldots, B_{J}$ in $\mathcal{F}_{S}$ such that $B=\bigcup_{j=1}^{J} B_{j}$ and $P\left[B_{j}\right] \leq \varepsilon$ for all $j=1, \ldots, J$. It follows from (3.2) that for all $j=1, \ldots, J$,

$$
Y^{0}+J \mathbf{1}_{B_{j}}\left(2 Z^{0}-Y^{0}\right) \in \bar{C}_{n_{0}},
$$

which together with the fact that $\bar{C}_{n_{0}}$ is convex, implies that

$$
2 Z^{0}=\frac{1}{J} \sum_{j=1}^{J}\left(Y^{0}+J \mathbf{1}_{B_{j}}\left(2 Z^{0}-Y^{0}\right)\right) \in \bar{C}_{n_{0}}
$$

Hence, there exists a sequence $\left\{Z^{k}\right\}_{k \geq 1}$ in $C_{n_{0}}$ such that $\left(Z^{k}-2 Z^{0}\right)^{*} \stackrel{P}{\rightarrow} 0$. Then, for all $k \geq 1, \tilde{Z}^{k}:=\left(Z^{k} \vee 2 Z^{0}\right)-2 Z^{0} \geq 0$ and $\left(\tilde{Z}^{k}\right)^{*} \stackrel{P}{\rightarrow} 0$. Since $\tilde{f}$ is concave and increasing,

$$
\tilde{f}\left(-\tilde{Z}^{k}\right) \leq 2 \tilde{f}\left(Z^{0}\right)-\tilde{f}\left(Z^{k} \vee 2 Z^{0}\right) \leq 2 \tilde{f}\left(Z^{0}\right)-\tilde{f}\left(Z^{k}\right) \leq-1 \quad \text { for all } k \geq 1 .
$$

By possibly passing to a subsequence, we can assume that for all $k \geq 1$,

$$
P\left[\left(\tilde{Z}^{k}\right)^{*}>2^{-2 k}\right]<2^{-k}
$$


Then, by the Borel-Cantelli Lemma, $Z:=\sum_{k \geq 1} 2^{k} \tilde{Z}^{k}$ is a well-defined process in $\mathcal{R}_{S, B}^{0}$, and we get for all $k \geq 1$,

$$
\tilde{f}(-Z) \leq \tilde{f}\left(-2^{k} \tilde{Z}^{k}\right) \leq 2^{k} \tilde{f}\left(-\tilde{Z}^{k}\right) \leq-2^{k},
$$

which contradicts $\tilde{f}(-Z) \in \mathbb{R}$. Hence, (3.1) must be true.

Corollary 3.2 If $\left(\Omega, \mathcal{F}_{0}, P\right)$ is atomless, then there exist no real-valued concave monetary utility functionals on $\mathcal{R}^{0}$.

Proof If $\phi: \mathcal{R}^{0} \rightarrow \mathbb{R}$ were a concave monetary utility functional, it would have to be constant by Theorem 3.1. But this cannot be.

Theorem 3.1 shows that it is a rather strong restriction to require coherent and concave utility functionals on $\mathcal{R}^{0}$ to be real-valued. Therefore, we allow them to take values in $[-\infty, \infty)$. If the coherent or concave monetary utility functional of a process $X \in \mathcal{R}^{0}$ is $-\infty$, this means that $X$ is so risky that no additional amount of money can make it acceptable.

Definition 3.3 We call a mapping $\phi: \mathcal{R}^{0} \rightarrow[-\infty, \infty)$ a concave monetary utility functional on $\mathcal{R}^{0}$ if

(0) $\phi(X) \in \mathbb{R}$, for all $X \in \mathcal{R}^{\infty}$

(1) $\phi(\lambda X+(1-\lambda) Y) \geq \lambda \phi(X)+(1-\lambda) \phi(Y)$, for all $X, Y \in \mathcal{R}^{0}$ and $\lambda \in[0,1]$

(2) $\phi(X) \leq \phi(Y)$, for all $X, Y \in \mathcal{R}^{0}$ such that $X \leq Y$

(3) $\phi(X+m)=\phi(X)+m$, for all $X \in \mathcal{R}^{0}$ and $m \in \mathbb{R}$

We call a concave monetary utility functional $\phi$ on $\mathcal{R}^{0}$ a coherent utility functional if it satisfies the additional property:

(4) $\phi(\lambda X)=\lambda \phi(X)$, for all $\lambda \in \mathbb{R}_{+}$.

We call the negative $\rho=-\phi$ of a concave monetary utility functional $\phi$ on $\mathcal{R}^{0}$, a convex monetary risk measure on $\mathcal{R}^{0}$. If $\phi$ is a coherent utility functional on $\mathcal{R}^{0}$, we call $\rho$ a coherent risk measure on $\mathcal{R}^{0}$.

Obviously, the restriction of a concave monetary utility functional $\phi$ on $\mathcal{R}^{0}$ to $\mathcal{R}^{\infty}$ is a concave monetary utility functional on $\mathcal{R}^{\infty}$. In the following we are going to investigate when a concave monetary utility functional on $\mathcal{R}^{\infty}$ can be extended to a concave monetary utility functional on $\mathcal{R}^{0}$.

Definition 3.4 For a function $\phi: \mathcal{R}^{\infty} \rightarrow \mathbb{R}$, we define its extension $\phi_{\mathrm{ext}}$ to $\mathcal{R}^{0}$ by

$$
\phi_{\mathrm{ext}}(X):=\lim _{n \rightarrow \infty} \lim _{m \rightarrow-\infty} \phi((X \wedge n) \vee m), \quad X \in \mathcal{R}^{0}
$$

It can easily be checked that the extension $\phi_{\text {ext }}$ of a concave monetary utility functional $\phi$ on $\mathcal{R}^{\infty}$ to $\mathcal{R}^{0}$ is an increasing, translation invariant function from $\mathcal{R}^{0}$ to $[-\infty, \infty]$. The following proposition shows that it is also concave. 
Proposition 3.5 Let $\phi: \mathcal{R}^{\infty} \rightarrow \mathbb{R}$ be increasing and concave. Then, with the convention $-\infty+\infty=-\infty$, also the extension $\phi_{\mathrm{ext}}: \mathcal{R}^{0} \rightarrow[-\infty, \infty]$ is concave.

Proof Let $X, Y \in \mathcal{R}^{0}$ and $\lambda \in(0,1)$. Set $\mu:=\lambda \wedge(1-\lambda)$. It can easily be checked that for all $n>0$ and $m<0$,

$$
\begin{aligned}
& \lambda[(X \wedge n) \vee m]+(1-\lambda)[(Y \wedge n) \vee m] \\
& \quad \leq[\lambda(X \wedge n)+(1-\lambda)(Y \wedge n)] \vee[n+\mu m] \\
& \quad \leq[(\lambda X+(1-\lambda) Y) \wedge n] \vee[n+\mu m] .
\end{aligned}
$$

Hence,

$$
\begin{aligned}
\phi_{\mathrm{ext}}(\lambda X+(1-\lambda) Y) & =\lim _{n \rightarrow \infty} \lim _{m \rightarrow-\infty} \phi([(\lambda X+(1-\lambda) Y) \wedge n] \vee m) \\
& =\lim _{n \rightarrow \infty} \lim _{m \rightarrow-\infty} \phi([(\lambda X+(1-\lambda) Y) \wedge n] \vee[n+\mu m]) \\
& \geq \lim _{n \rightarrow \infty} \lim _{m \rightarrow-\infty} \phi(\lambda[(X \wedge n) \vee m]+(1-\lambda)[(Y \wedge n) \vee m]) \\
& \geq \lim _{n \rightarrow \infty} \lim _{m \rightarrow-\infty}\{\lambda \phi([X \wedge n] \vee m)+(1-\lambda) \phi([Y \wedge n] \vee m)\} \\
& =\lambda \phi_{\text {ext }}(X)+(1-\lambda) \phi_{\text {ext }}(Y) .
\end{aligned}
$$

It follows from Proposition 3.5 and the three lines before it that the extension $\phi_{\text {ext }}$ of a concave monetary utility functional $\phi$ on $\mathcal{R}^{\infty}$ is a concave monetray utility functional on $\mathcal{R}^{0}$, if and only if $\phi_{\text {ext }}(X)<\infty$ for all $X \in \mathcal{R}^{0}$.

Note that $\phi_{\text {ext }}$ need not be the only possible extension of a concave monetary utility functional $\phi$ from $\mathcal{R}^{\infty}$ to $\mathcal{R}^{0}$, that is, it is possible that there exists a concave monetary utility functional $\psi$ on $\mathcal{R}^{0}$ such that $\psi=\phi$ on $\mathcal{R}^{\infty}$ but $\psi \neq \phi_{\text {ext }}$ on $\mathcal{R}^{0}$.

In the following we will focus our attention on the extension of concave monetary utility functionals $\phi$ on $\mathcal{R}^{\infty}$ that are continuous for bounded decreasing sequences and, by Theorem 2.3, can therefore be represented as

$$
\phi(X)=\inf _{a \in \mathcal{D}_{\sigma}}\{\langle X, a\rangle-\gamma(a)\}, \quad X \in \mathcal{R}^{\infty},
$$

for a penalty function $\gamma: \mathcal{D}_{\sigma} \rightarrow[-\infty, \infty)$.

Definition 3.6 For a penalty function $\gamma: \mathcal{D}_{\sigma} \rightarrow[-\infty, \infty)$ and a constant $K \in \mathbb{R}$, we denote

$$
\mathcal{Q}_{\sigma}^{\gamma, K}:=\left\{a \in \mathcal{D}_{\sigma} \mid \gamma(a) \geq-K\right\} \quad \text { and } \quad\left\langle\mathcal{Q}_{\sigma}^{\gamma, K}\right|:={\overline{\operatorname{conv}\left(\mathcal{Q}_{\sigma}^{\gamma, K}\right)}}^{\sigma\left(\mathcal{A}^{1}, \mathcal{R}^{\infty}\right)},
$$

where $\overline{\operatorname{conv}\left(\mathcal{Q}_{\sigma}^{\gamma, K}\right)}{ }^{\sigma\left(\mathcal{A}^{1}, \mathcal{R}^{\infty}\right)}$ is the $\sigma\left(\mathcal{A}^{1}, \mathcal{R}^{\infty}\right)$-closure of the convex hull of $\mathcal{Q}_{\sigma}^{\gamma, K}$. Furthermore,

$$
\mathcal{Q}_{\sigma}^{\gamma}:=\bigcup_{K>0} \mathcal{Q}_{\sigma}^{\gamma, K}=\left\{a \in \mathcal{D}_{\sigma} \mid \gamma(a)>-\infty\right\} \text { and }\left\langle\mathcal{Q}_{\sigma}^{\gamma}\right\rangle:=\overline{\operatorname{conv}\left(\mathcal{Q}_{\sigma}^{\gamma}\right)}{ }^{\sigma\left(\mathcal{A}^{1}, \mathcal{R}^{\infty}\right)}
$$


Remark 3.7 If $\gamma$ is concave and $\sigma\left(\mathcal{A}^{1}, \mathcal{R}^{\infty}\right)$-upper semicontinuous, then for all $K \in \mathbb{R}$

$$
\left\langle\mathcal{Q}_{\sigma}^{\gamma, K}\right\rangle=\mathcal{Q}_{\sigma}^{\gamma, K}
$$

Note that $\phi^{*}$ is always concave and $\sigma\left(\mathcal{A}^{1}, \mathcal{R}^{\infty}\right)$-upper semicontinuous.

Proposition 3.8 Let $\gamma_{1}, \gamma_{2}: \mathcal{D}_{\sigma} \rightarrow[-\infty, \infty)$ be two penalty functions that induce the same concave monetary utility functional on $\mathcal{R}^{\infty}$, that is,

$$
\inf _{a \in \mathcal{D}_{\sigma}}\left\{\langle X, a\rangle-\gamma_{1}(a)\right\}=\inf _{a \in \mathcal{D}_{\sigma}}\left\{\langle X, a\rangle-\gamma_{2}(a)\right\}, \quad \text { for all } X \in \mathcal{R}^{\infty} .
$$

Then, $\left\langle\mathcal{Q}_{\sigma}^{\gamma_{1}}\right\rangle=\left\langle\mathcal{Q}_{\sigma}^{\gamma_{2}}\right\rangle$.

Proof Suppose that $\left\langle\mathcal{Q}_{\sigma}^{\gamma_{1}}\right\rangle \neq\left\langle\mathcal{Q}_{\sigma}^{\gamma_{2}}\right\rangle$. By symmetry, we can assume that there exists an $a \in \mathcal{Q}_{\sigma}^{\gamma_{1}}$ such that $a \notin\left\langle\mathcal{Q}_{\sigma}^{\gamma_{2}}\right\rangle$. Since $\left\langle\mathcal{Q}_{\sigma}^{\gamma_{2}}\right\rangle$ is $\sigma\left(\mathcal{A}^{1}, \mathcal{R}^{\infty}\right)$-closed and convex, the separating hyperplane theorem yields an $X \in \mathcal{R}^{\infty}$, such that

$$
\langle X, a\rangle<\inf _{b \in\left\langle\mathcal{Q}_{\sigma}^{\gamma_{2}}\right\rangle}\langle X, b\rangle .
$$

Note that $\sup _{b \in \mathcal{Q}_{\sigma}^{\gamma}} \gamma_{2}(b)<\infty$ and $\gamma_{1}(a) \in \mathbb{R}$. Therefore, there exists a $\lambda>0$ such that

$$
\langle\lambda X, a\rangle+\sup _{b \in \mathcal{Q}_{\sigma}^{\gamma_{2}}} \gamma_{2}(b)-\gamma_{1}(a)<\inf _{b \in\left\langle\mathcal{Q}_{\sigma}^{\gamma_{2}}\right\rangle}\langle\lambda X, b\rangle,
$$

and therefore,

$$
\begin{aligned}
\phi(\lambda X) & =\inf _{b \in \mathcal{Q}_{\sigma}^{\gamma_{2}}}\left\{\langle\lambda X, b\rangle-\gamma_{2}(b)\right\} \geq \inf _{b \in\left\langle\mathcal{Q}_{\sigma}^{\gamma_{2}}\right\rangle}\langle\lambda X, b\rangle-\sup _{b \in \mathcal{Q}_{\sigma}^{\gamma_{2}}} \gamma_{2}(b) \\
& >\langle\lambda X, a\rangle-\gamma_{1}(a) \geq \inf _{b \in \mathcal{Q}_{\sigma}^{\gamma_{1}}}\left\{\langle\lambda X, b\rangle-\gamma_{1}(b)\right\}=\phi(\lambda X)
\end{aligned}
$$

which is absurd.

Theorem 3.9 Let $\phi$ be a concave monetary utility functional on $\mathcal{R}^{\infty}$ of the form (3.3). Then the following five properties are equivalent:

(1) $\phi_{\text {ext }}(X)<\infty$ for all $X \in \mathcal{R}^{0}$.

(2) There exists a $\delta>0$ such that for every $[0, T]$-valued stopping time $S$ and all $B \in \mathcal{F}_{S}$ with $P[B] \leq \delta$, there exists a $K>0$ such that

$$
\inf _{a \in \mathcal{Q}_{\sigma}^{\gamma, K}}\left\langle\mathbf{1}_{B} \mathbf{1}_{[S, T]}, a\right\rangle=0 .
$$


(3) There exists a $K>0$ such that for every [0,T]-valued stopping time $S$ and all $B \in \mathcal{F}_{S}$ with $P[B] \leq K^{-1}$,

$$
\inf _{a \in \mathcal{Q}_{\sigma}^{\gamma, K}}\left\langle\mathbf{1}_{B} \mathbf{1}_{[S, T]}, a\right\rangle=0
$$

(4) There exists a $\delta>0$ such that for every $[0, T]$-valued stopping time $S$ and all $B \in \mathcal{F}_{S}$ with $P[B] \leq \delta$, there exists a $K>0$ and an $a \in\left\langle\mathcal{Q}_{\sigma}^{\gamma, K}\right\rangle$ such that

$$
\left\langle\mathbf{1}_{B} \mathbf{1}_{[S, T]}, a\right\rangle=0 \quad \text { and } \quad \operatorname{Var}\left(a^{\mathrm{pr}}\right)+\operatorname{Var}\left(a^{\mathrm{op}}\right) \leq K
$$

(5) There exists a $K>0$, such that for every [0,T]-valued stopping time $S$ and all $B \in \mathcal{F}_{S}$ with $P[B] \leq K^{-1}$, there exists an $a \in\left\langle\mathcal{Q}_{\sigma}^{\gamma, K}\right\rangle$ such that

$$
\left\langle\mathbf{1}_{B} \mathbf{1}_{[S, T]}, a\right\rangle=0 \quad \text { and } \quad \operatorname{Var}\left(a^{\mathrm{pr}}\right)+\operatorname{Var}\left(a^{\mathrm{op}}\right) \leq K
$$

It is obvious that if a function $\phi: \mathcal{R}^{\infty} \rightarrow \mathbb{R}$ satisfies condition (4) of Definition 2.1 , then so does $\phi_{\text {ext }}$. Moreover, if $\phi$ is a coherent utility functional on $\mathcal{R}^{\infty}$ with representation

$$
\phi(X):=\inf _{a \in \mathcal{Q}_{\sigma}}\langle X, a\rangle, \quad X \in \mathcal{R}^{\infty}
$$

for some subset $\mathcal{Q}_{\sigma} \subset \mathcal{D}_{\sigma}$, then $\phi^{*}$ on $\mathcal{D}_{\sigma}$ is given by

$$
\phi^{*}(a)=\left\{\begin{array}{cc}
0 & \text { if } a \in\left\langle\mathcal{Q}_{\sigma}\right\rangle \\
-\infty & \text { if } a \in \mathcal{D}_{\sigma} \backslash\left\langle\mathcal{Q}_{\sigma}\right\rangle
\end{array} .\right.
$$

Hence, the following is an immediate consequence of Theorem 3.9.

Corollary 3.10 Let $\phi$ be a coherent utility functional on $\mathcal{R}^{\infty}$ with representation (3.4). Then, the following four properties are equivalent:

(1) $\phi_{\text {ext }}(X)<\infty$ for all $X \in \mathcal{R}^{0}$.

(2) There exists a $\delta>0$ such that for every [0,T]-valued stopping time $S$ and all $B \in \mathcal{F}_{S}$ with $P[B] \leq \delta$, we have

$$
\inf _{a \in \mathcal{Q}_{\sigma}}\left\langle\mathbf{1}_{B} \mathbf{1}_{[S, T]}, a\right\rangle=0
$$

(3) There exists a $\delta>0$, such that for every $[0, T]$-valued stopping time $S$ and all $B \in \mathcal{F}_{S}$ with $P[B] \leq \delta$, there exists $a K>0$ and an $a \in\left\langle\mathcal{Q}_{\sigma}\right\rangle$ such that

$$
\left\langle\mathbf{1}_{B} \mathbf{1}_{[S, T]}, a\right\rangle=0 \quad \text { and } \quad \operatorname{Var}\left(a^{\mathrm{pr}}\right)+\operatorname{Var}\left(a^{\mathrm{op}}\right) \leq K
$$


(4) There exists a $K>0$, such that for every [0,T]-valued stopping time $S$ and all $B \in \mathcal{F}_{S}$ with $P[B] \leq K^{-1}$, there exists an $a \in\left\langle\mathcal{Q}_{\sigma}\right\rangle$ such that

$$
\left\langle\mathbf{1}_{B} \mathbf{1}_{[S, T]}, a\right\rangle=0 \quad \text { and } \quad \operatorname{Var}\left(a^{\mathrm{pr}}\right)+\operatorname{Var}\left(a^{\mathrm{op}}\right) \leq K
$$

\section{Proof of Theorem 3.9}

In the proof of Theorem 3.9 we will make use of the subsequent lemma. In the proof of the lemma we need the spaces $\hat{\mathcal{R}}^{p}$ and $\hat{\mathcal{A}}^{q}$ that are defined like $\mathcal{R}^{p}$ and $\mathcal{A}^{q}$ but with respect to the filtration $\left(\hat{\mathcal{F}}_{t}\right)_{t \in[0, T]}$, given by $\hat{\mathcal{F}}_{t}:=\mathcal{F}$ for all $t \in[0, T]$. By $\Pi_{\text {op }}$ we denote the optional projection from $\hat{\mathcal{R}}^{\infty}$ to $\mathcal{R}^{\infty}$. Theorem VI.47 on p. 108 in Dellacherie and Meyer [10] guarantees that the optional projection of a measurable, bounded, càdlàg process is again càdlàg. The dual projection $\Pi^{*}: \hat{\mathcal{A}}^{1} \rightarrow \mathcal{A}^{1}$ is defined as follows: for $a=\left(a^{1}, a^{\mathrm{r}}\right) \in \hat{\mathcal{A}}^{1}$ let $\tilde{a}^{\mathrm{l}}$ be the dual predictable projection of $a^{1}$ with respect to the filtration $\left(\mathcal{F}_{t}\right)$ and $\tilde{a}^{\mathrm{r}}$ the dual optional projection of $a^{\mathrm{r}}$. The process $\tilde{a}^{\mathrm{r}}$ can be split into a purely discontinuous, optional finite variation process $\tilde{a}^{\mathrm{d}}$ and a continuous finite variation process $\tilde{a}^{\mathrm{c}}$ such that $\tilde{a}_{0}^{\mathrm{c}}=0$. We set $\Pi^{*} a:=\left(\tilde{a}^{1}+\tilde{a}^{\mathrm{c}}, \tilde{a}^{\mathrm{d}}\right)$. Then $\left\langle X, \Pi^{*} a\right\rangle=\langle X, a\rangle$ for all $X \in \mathcal{R}^{\infty}$ (see Cheridito et al. [5]).

Lemma 4.1 Let $K$ be a positive constant, $S$ a $[0, T]$-valued stopping time and $B \in \mathcal{F}_{S}$. Then the set

$$
H_{K, S, B}=\left\{a \in \mathcal{A}^{1} \mid\left\langle\mathbf{1}_{B} \mathbf{1}_{[S, T]}, a\right\rangle=0, \operatorname{Var}\left(a^{\mathrm{pr}}\right)+\operatorname{Var}\left(a^{\mathrm{op}}\right) \leq K\right\}
$$

is $\sigma\left(\mathcal{A}^{1}, \mathcal{R}^{\infty}\right)$-compact.

Proof It follows from Theorem VII.67 on p. 255 of Dellacherie and Meyer [10] (see also Variant (a) of Theorem VII.2 on p. 189 of [10]) that $\hat{\mathcal{A}}^{\infty}$ is the dual space of $\hat{\mathcal{R}}^{1}$. Therefore, Alaoglu's Theorem implies that $\left\{a \in \hat{\mathcal{A}}^{\infty} \mid \operatorname{Var}\left(a^{\mathrm{pr}}\right)\right.$ $\left.+\operatorname{Var}\left(a^{\mathrm{op}}\right) \leq K\right\}$ is $\sigma\left(\hat{\mathcal{A}}^{\infty}, \hat{\mathcal{R}}^{1}\right)$-compact. The image $\left\{a \in \hat{\mathcal{A}}^{1} \mid \operatorname{Var}\left(a^{\mathrm{pr}}\right)\right.$ $\left.+\operatorname{Var}\left(a^{\mathrm{op}}\right) \leq K\right\} \quad$ of $\quad\left\{a \in \hat{\mathcal{A}}^{\infty} \mid \operatorname{Var}\left(a^{\mathrm{pr}}\right)+\operatorname{Var}\left(a^{\mathrm{op}}\right) \leq K\right\} \quad$ under the $\sigma\left(\hat{\mathcal{A}}^{\infty}, \hat{\mathcal{R}}^{1}\right) / \sigma\left(\hat{\mathcal{A}}^{1}, \hat{\mathcal{R}}^{\infty}\right)$-continuous map id $: \hat{\mathcal{A}}^{\infty} \rightarrow \hat{\mathcal{A}}^{1}$ is $\sigma\left(\hat{\mathcal{A}}^{1}, \hat{\mathcal{R}}^{\infty}\right)$-compact. It can easily be checked that the dual projection $\Pi^{*}: \hat{\mathcal{A}}^{1} \rightarrow \mathcal{A}^{1}$ discussed above is $\sigma\left(\hat{\mathcal{A}}^{1}, \hat{\mathcal{R}}^{\infty}\right) / \sigma\left(\mathcal{A}^{1}, \mathcal{R}^{\infty}\right)$-continuous. Hence, the image

$\tilde{H}_{K}:=\left\{\Pi^{*} a \mid a \in \hat{\mathcal{A}}^{1}, \operatorname{Var}\left(a^{\mathrm{pr}}\right)+\operatorname{Var}\left(a^{\mathrm{op}}\right) \leq K\right\} \quad$ of $\quad\left\{a \in \hat{\mathcal{A}}^{1} \mid \operatorname{Var}\left(a^{\mathrm{pr}}\right)\right.$ $\left.+\operatorname{Var}\left(a^{\mathrm{op}}\right) \leq K\right\}$ under $\Pi^{*}$ is $\sigma\left(\mathcal{A}^{1}, \mathcal{R}^{\infty}\right)$-compact. Now, let $\left(b^{\lambda}\right)_{\lambda \in \Lambda}$ be a net in $H_{K, S, B}$ and $b \in \mathcal{A}^{1}$ such that

$$
b^{\lambda} \rightarrow b \text { in } \sigma\left(\mathcal{A}^{1}, \mathcal{R}^{\infty}\right)
$$


Obviously, for all $X \in \hat{\mathcal{R}}^{\infty}$,

$$
\left\langle X, b^{\lambda}-b\right\rangle=\left\langle\Pi_{\mathrm{op}} X, b^{\lambda}-b\right\rangle \rightarrow 0,
$$

that is, $b^{\lambda} \rightarrow b$ also in $\sigma\left(\hat{\mathcal{A}}^{1}, \hat{\mathcal{R}}^{\infty}\right)$. Since $\hat{\mathcal{R}}^{\infty}$ separates points in $\hat{\mathcal{A}}^{1}$, the $\sigma\left(\hat{\mathcal{A}}^{1}, \hat{\mathcal{R}}^{\infty}\right)$-compact set $\left\{a \in \hat{\mathcal{A}}^{1} \mid \operatorname{Var}\left(a^{\mathrm{pr}}\right)+\operatorname{Var}\left(a^{\mathrm{op}}\right) \leq K\right\}$ is closed in $\hat{\mathcal{A}}^{1}$. Therefore, $\operatorname{Var}\left(b^{\mathrm{pr}}\right)+\operatorname{Var}\left(b^{\mathrm{op}}\right) \leq K$. Moreover, it follows from (4.1) that $\left\langle\mathbf{1}_{B} \mathbf{1}_{[S, T]}, b\right\rangle=0$. Hence, $b \in H_{K, S, B}$. This shows that $H_{K, S, B}$ is a $\sigma\left(\mathcal{A}^{1}, \mathcal{R}^{\infty}\right)$ closed subset of the $\sigma\left(\mathcal{A}^{1}, \mathcal{R}^{\infty}\right)$-compact set $\tilde{H}_{K}$, which implies the assertion of the lemma.

Proof of Theorem 3.9 (1) $\Rightarrow$ (3): If there is no $K>0$ satisfying (3), there exists for every $j \geq 1$, a $[0, T]$-valued stopping time $S_{j}$ and $B_{j} \in \mathcal{F}_{S_{j}}$ such that

$$
P\left[B_{j}\right] \leq 2^{-j} \quad \text { and } \quad \varepsilon_{j}:=\inf _{a \in \mathcal{Q}_{\sigma}^{\gamma, j}}\left\langle\mathbf{1}_{B_{j}} \mathbf{1}_{\left[S_{j}, T\right]}, a\right\rangle>0
$$

The Borel-Cantelli Lemma guarantees that

$$
X:=\sum_{j \geq 1} \frac{j}{\varepsilon_{j}} \mathbf{1}_{B_{j}} \mathbf{1}_{\left[S_{j}, T\right]}
$$

is a well-defined process in $\mathcal{R}^{0}$. Denote $\bar{\gamma}:=\sup _{a \in \mathcal{Q}_{\sigma}^{\gamma}} \gamma(a)<\infty$, fix $j$ and set $n=j / \varepsilon_{j}$. Then,

$$
\begin{aligned}
\phi(X \wedge n) & \geq \inf _{a \in \mathcal{Q}_{\sigma}^{\gamma}}\left\{\left\langle\frac{j}{\varepsilon_{j}} 1_{B_{j}} 1_{\left[S_{j}, T\right]}, a\right\rangle-\gamma(a)\right\} \\
& =\min \left\{\inf _{a \in \mathcal{Q}_{\sigma}^{\gamma, j}}\left\langle\frac{j}{\varepsilon_{j}} 1_{B_{j}} 1_{\left[S_{j}, T\right]}, a\right\rangle-\gamma(a) ; \inf _{a \in \mathcal{Q}_{\sigma}^{\gamma} \backslash \mathcal{Q}_{\sigma}^{\gamma, j}}\left\langle\frac{j}{\varepsilon_{j}} 1_{B_{j}} 1_{\left[S_{j}, T\right]}, a\right\rangle-\gamma(a)\right\} \\
& \geq \min \{j-\bar{\gamma} ; j\},
\end{aligned}
$$

and it follows that

$$
\phi_{\mathrm{ext}}(X)=\lim _{n \rightarrow \infty} \phi(X \wedge n)=\infty
$$

which contradicts (1).

$(3) \Rightarrow(5)$ : Choose a $K>0$ that fulfils (3) and set $\tilde{K}=2 K$. Let $S$ be a $[0, T]-$ valued stopping time and $B \in \mathcal{F}_{S}$ such that $P[B] \leq \tilde{K}^{-1}$. By Lemma 4.1, the convex set

$$
H_{\tilde{K}, S, B}:=\left\{a \in \mathcal{A}^{1} \mid\left\langle\mathbf{1}_{B} \mathbf{1}_{[S, T]}, a\right\rangle=0, \operatorname{Var}\left(a^{\mathrm{pr}}\right)+\operatorname{Var}\left(a^{\mathrm{op}}\right) \leq \tilde{K}\right\},
$$


is $\sigma\left(\mathcal{A}^{1}, \mathcal{R}^{\infty}\right)$-compact. Assume that $H_{\tilde{K}, S, B}$ and the convex, $\sigma\left(\mathcal{A}^{1}, \mathcal{R}^{\infty}\right)$-closed $\operatorname{set}\left\langle\mathcal{Q}_{\sigma}^{\gamma, \tilde{K}}\right\rangle$ are disjoint. Then, it follows from the separating hyperplane theorem that there exists an $X \in \mathcal{R}^{\infty}$ such that

$$
\sup _{a \in H_{\tilde{K}, S, B}}\langle X, a\rangle<\inf _{a \in\left\langle\mathcal{Q}_{\sigma}^{\gamma, \tilde{K}}\right\rangle}\langle X, a\rangle
$$

Modify the process $X$ as follows:

$$
\tilde{X}_{t}:= \begin{cases}X_{t}, & t<S \\ X_{t} & \text { on } B^{c} \text { for } t \geq S \\ 0 & \text { on } B \text { for } t \geq S\end{cases}
$$

Let $\Theta$ be the set of all stopping times with values in $[0, T]$ and set

$$
M:=\sup _{\theta \in \Theta}\left\|\tilde{X}_{\theta}\right\|_{1}
$$

For every $\varepsilon>0$, there exists a $\theta \in \Theta$ such that $\left\|\tilde{X}_{\theta}\right\|_{1}>M-\varepsilon$. Define

$$
b_{t}:= \begin{cases}\left(0, \tilde{K} \mathbf{1}_{\{t \geq \theta\}}\right) & \text { for } \tilde{X}_{\theta} \geq 0 \\ \left(0,-\tilde{K} \mathbf{1}_{\{t \geq \theta\}}\right) & \text { for } \tilde{X}_{\theta}<0\end{cases}
$$

and

$$
\tilde{b}_{t}:= \begin{cases}b_{t}, & t<S \\ b_{t} & \text { on } B^{c} \text { for } t \geq S . \\ b_{S-} & \text { on } B \text { for } t \geq S\end{cases}
$$

This yields for the left-hand side of (4.2),

$$
\sup _{a \in H_{\tilde{K}, S, B}}\langle X, a\rangle \geq\langle X, \tilde{b}\rangle=\langle\tilde{X}, \tilde{b}\rangle=\tilde{K}\left\|\tilde{X}_{\theta}\right\|_{1}>\tilde{K}(M-\varepsilon),
$$

and therefore,

$$
\sup _{a \in H_{\tilde{K}, S, B}}\langle X, a\rangle \geq \tilde{K} M
$$

To obtain a contradiction, we introduce the stopping time

$$
\tau:=\inf \left\{t \geq 0|| X_{t} \mid \geq \tilde{K} M\right\} \quad \text { with } \quad \inf \emptyset=\infty
$$


By Markov’s inequality,

$$
\begin{aligned}
P[\tau \leq T] & =P\left[\{\tau \leq T\} \cap\left\{\left|X_{\tau}\right| \geq \tilde{K} M\right\}\right] \\
& \leq P\left[\{\tau \leq T\} \cap\left\{\left|\tilde{X}_{\tau}\right| \geq \tilde{K} M\right\}\right]+\tilde{K}^{-1} \\
& \leq \tilde{K}^{-1} \frac{\left\|\tilde{X}_{\tau}\right\|_{1}}{M}+\tilde{K}^{-1} \leq 2 \tilde{K}^{-1}=K^{-1}
\end{aligned}
$$

Hence, it follows from (3) that

$$
\inf _{a \in\left\langle\mathcal{Q}_{\sigma}^{\gamma, \tilde{K}}\right\rangle}\left\langle X \mathbf{1}_{[\tau, T]}, a\right\rangle \leq\|X\|_{\mathcal{R}^{\infty}} \inf _{a \in \mathcal{Q}_{\sigma}^{\gamma, K}}\left\langle\mathbf{1}_{[\tau, T]}, a\right\rangle=0
$$

and we obtain for the right hand side of (4.2),

$$
\inf _{a \in\left\langle\mathcal{Q}_{\sigma}^{\gamma, \tilde{K}}\right\rangle}\langle X, a\rangle \leq \sup _{a \in\left\langle\mathcal{Q}_{\sigma}^{\gamma, \tilde{K}}\right\rangle}\left\langle X \mathbf{1}_{[0, \tau)}, a\right\rangle+\inf _{a \in\left\langle\mathcal{Q}_{\sigma}^{\gamma, \tilde{K}}\right\rangle}\left\langle X \mathbf{1}_{[\tau, T]}, a\right\rangle \leq \tilde{K} M .
$$

This is in contradiction to (4.2) and (4.3). Therefore, $H_{\tilde{K}, S, B} \cap\left\langle\mathcal{Q}_{\sigma}^{\gamma, \tilde{K}}\right\rangle \neq \emptyset$, which proves (5).

$(5) \Rightarrow(4)$ is trivial.

$(4) \Rightarrow(2)$ : Assume (4) holds with $\delta>0$ and let $S$ be a $[0, T]$-valued stopping time and $B \in \mathcal{F}_{S}$ such that $P[B] \leq \delta$. Then, there exists a $K>0$ and an $a \in\left\langle\mathcal{Q}_{\sigma}^{\gamma, K}\right\rangle$ such that

$$
\left\langle 1_{B} 1_{[S, T]}, a\right\rangle=0 .
$$

Hence, for all $\varepsilon>0$, there exists a convex combination $\sum_{j=1}^{J} \lambda_{j} a_{j}$ of elements $a_{1}, \ldots, a_{J}$ in $\mathcal{Q}_{\sigma}^{\gamma, K}$ such that

$$
\left\langle 1_{B} 1_{[S, T]}, \sum_{j=1}^{J} \lambda_{j} a_{j}\right\rangle \leq \varepsilon
$$

which implies that for at least one of the $a_{j}$ 's,

$$
\left\langle 1_{B} 1_{[S, T]}, a_{j}\right\rangle \leq \varepsilon
$$

This proves (2).

(2) $\Rightarrow$ (1): Assume that (2) holds for $\delta>0$ and let $X \in \mathcal{R}^{0}$. Let $N>0$ be so large that $P\left[X^{*} \geq N\right] \leq \delta$, and introduce the stopping time

$$
\tau:=\inf \left\{t|| X_{t} \mid \geq N\right\} \quad \text { with the convention } \quad \inf \emptyset=\infty \text {. }
$$


Then, $P[\tau \leq T] \leq \delta$. By assumption, there exists a $K>0$ such that

$$
\inf _{a \in \mathcal{Q}_{\sigma}^{\gamma, K}}\left\langle\mathbf{1}_{[\tau, T]}, a\right\rangle=0
$$

Hence, for all $n>0$ and $m<0$,

$$
\begin{aligned}
& \inf _{a \in \mathcal{Q}_{\sigma}^{\gamma}}\{\langle(X \wedge n) \vee m, a\rangle-\gamma(a)\} \\
& \quad \leq \inf _{a \in \mathcal{Q}_{\sigma}^{\gamma, K}}\left\{\left\langle\left(1_{[0, \tau)} X \wedge n\right) \vee m, a\right\rangle+\left\langle\left(1_{[\tau, T]} X \wedge n\right) \vee m, a\right\rangle-\gamma(a)\right\} \\
& \quad \leq N+K .
\end{aligned}
$$

\section{Examples}

5.1 The risk measure $\mathrm{AVaR}_{\alpha}\left(\inf _{0 \leq t \leq T} X_{t}\right)$ and its application to the Cramér-Lundberg process

Assume that the riskless interest rate is zero and the surplus of an insurance company resulting from incoming premia payments and settlements of claims is modelled with an adapted càdlàg process $\left(X_{t}\right)_{t \geq 0}$. One of the concepts that has received a lot of attention in insurance mathematics is the ruin probability (see for instance Asmussen [4] or Rolski et al. [17] and the references therein). Denote for $m \in \mathbb{R}$ and $T>0$,

$$
\psi(m, X, T):=P\left[m+\inf _{t \in[0, T]} X_{t}<0\right]
$$

and

$$
\psi(m, X):=P\left[m+\inf _{t \geq 0} X_{t}<0\right]=\lim _{T \rightarrow \infty} \psi(m, X, T)
$$

Note that for $\alpha \in(0,1)$,

$$
\inf \{m \mid \psi(m, X, T) \leq \alpha\}=\operatorname{VaR}_{\alpha}\left(\inf _{t \in[0, T]} X_{t}\right)
$$

and

$$
\inf \{m \mid \psi(m, X) \leq \alpha\}=\operatorname{VaR}_{\alpha}\left(\inf _{t \geq 0} X_{t}\right)
$$

where $\operatorname{VaR}_{\alpha}$ denotes value at risk at the level $\alpha$, given by

$$
\operatorname{VaR}_{\alpha}(Z):=\inf \{m \mid P[m+Z<0] \leq \alpha\}, \quad Z \in L^{0} .
$$


If $X$ is equal to the Cramér-Lundberg process

$$
C_{t}=c t-\sum_{j=1}^{N_{t}} Y_{j}
$$

where $c>0,\left(N_{t}\right)_{t \geq 0}$ is a Poisson process with intensity $\lambda>0$ and $\left\{Y_{j}\right\}_{j \geq 1}$ are positive random variables that are i.i.d., independent of $\left(N_{t}\right)_{t \geq 0}$ and such that there exists an $R>0$ with

$$
\lambda\left(\mathrm{E}\left[\exp \left(R Y_{1}\right)-1\right]\right)=c R
$$

then $\psi(m, X)$ can be estimated from above as follows: It can easily be checked that the process $M_{t}:=\left(e^{-R C_{t}}\right)_{t \geq 0}$ is a positive martingale. Introduce the stopping time

$$
\tau_{m}:=\inf \left\{t \geq 0 \mid m+C_{t}<0\right\}, \quad \text { with the convention inf } \emptyset:=\infty
$$

It follows from the optional sampling theorem that

$$
\begin{aligned}
\psi(m, C) & =P\left[\tau_{m}<\infty\right]<\mathrm{E}\left[\exp \left\{-R\left(m+C_{\tau_{m}}\right)\right\} 1_{\left\{\tau_{m}<\infty\right\}}\right] \\
& =\lim _{t \rightarrow \infty} \mathrm{E}\left[\exp \left\{-R\left(m+C_{\tau_{m} \wedge t}\right)\right\} 1_{\left\{\tau_{m} \leq t\right\}}\right] \\
& \leq \lim _{t \rightarrow \infty} \mathrm{E}\left[\exp \left\{-R\left(m+C_{\tau_{m} \wedge t}\right)\right\}\right] \\
& =\exp (-R m) .
\end{aligned}
$$

This implies that for all $T>0$,

$$
\operatorname{VaR}_{\alpha}\left(\inf _{t \in[0, T]} C_{t}\right) \leq \operatorname{VaR}_{\alpha}\left(\inf _{t \geq 0} C_{t}\right) \leq-\frac{\log \alpha}{R}
$$

For certain distributions of the $Y_{j}$ 's, $\psi(m, C)$ can even be calculated exactly. Since $\left(C_{t}\right)_{t \geq 0}$ is a strong Markov process, $\psi(m, C)$ satisfies the integral equation

$$
\frac{c}{\lambda}(\psi(m, C)-\psi(0, C))=\int_{0}^{m}[\psi(m-u, C)-1][1-G(u)] \mathrm{d} u
$$

where $G$ is the cumulative distribution function of $Y_{1}$ (see, for instance, equation 5.3.10 in Rolski et al. [17]). If $Y_{1}$ is exponentially distributed with parameter $\gamma>\lambda / c, \psi(m, C)$ can be calculated as

$$
\psi(m, C)=\frac{\lambda}{\gamma c} \exp \left\{-\left(\gamma-\frac{\lambda}{c}\right) m\right\}
$$


Hence, for all $T>0$,

$$
\operatorname{VaR}_{\alpha}\left(\inf _{t \in[0, T]} C_{t}\right) \leq \operatorname{VaR}_{\alpha}\left(\inf _{t \geq 0} C_{t}\right)=-\frac{\log \left(\frac{\gamma c}{\lambda} \alpha\right)}{\gamma-\frac{\lambda}{c}}
$$

However, value at risk has two major shortcomings. First, it does not take into account the size of the loss if a ruin occurs. Secondly, even as a risk measure on $L^{\infty}$, it is not subadditive (see Artzner et al. [2]), nor convex (see Föllmer and Schied [14]). That value at risk is not subadditive can be a problem if $X$ is a sum $X=\sum_{j=1}^{n} X^{j}$ of other processes and one wants to measure the risk contributions of the $X_{j}$ 's to the risk of $X$ (see for instance, Delbaen [8]).

An alternative to value at risk is average value at risk (also called conditional value at risk). The average value at risk at the level $\alpha \in(0,1)$ is given by

$$
\operatorname{AVaR}_{\alpha}(Z):=\frac{1}{\alpha} \int_{0}^{\alpha} \operatorname{VaR}_{u}(Z) \mathrm{d} u, \quad Z \in L^{0}
$$

It can be shown (see Föllmer and Schied [14]) that for all $Z \in L^{\infty}$,

$$
\operatorname{AVaR}_{\alpha}(Z)=-\phi(Z)
$$

where $\phi$ is the coherent utility functional on $L^{\infty}$ given by

$$
\phi(Z):=\inf _{Q \in \mathcal{P}_{\sigma}} \mathrm{E}_{Q}[Z]
$$

with $\mathcal{P}_{\sigma}:=\left\{Q \ll P \mid \frac{d Q}{d P} \leq \frac{1}{\alpha}\right\}$. It is easy to see that $\mathcal{P}_{\sigma}$ satisfies condition (2) of Corollary 3.10 (remember that for $T=0, \mathcal{R}^{\infty}=L^{\infty}$ and $\mathcal{R}^{0}=L^{0}$ ). Hence, $\phi_{\text {ext }}$ is a coherent utility functional on $L^{0}$. It can easily be checked that

$$
\phi_{\mathrm{ext}}(Z)=-\operatorname{AVaR}_{\alpha}(Z) \text { for all } Z \in L^{0} .
$$

Hence, $\mathrm{AVaR}_{\alpha}$ is a coherent risk measure on $L^{0}$. From there it is easy to see that

$$
X \mapsto \operatorname{AVaR}_{\alpha}\left(\inf _{t \in[0, T]} X_{t}\right)
$$

is a coherent risk measure on $\mathcal{R}^{0}$. For the classical Cramér-Lundberg process $\left(C_{t}\right)_{t \geq 0}$ we get from (5.4) that for all $T \geq 0$,

$$
\operatorname{AVaR}_{\alpha}\left(\inf _{t \in[0, T]} C_{t}\right) \leq \operatorname{AVaR}_{\alpha}\left(\inf _{t \geq 0} C_{t}\right) \leq \frac{1}{\alpha} \int_{0}^{\alpha}-\frac{\log u}{R} \mathrm{~d} u=\frac{1-\log \alpha}{R}
$$


If $Y_{1}$ is exponentially distributed with parameter $\gamma>\lambda / c$, then by (5.5), for all $T>0$,

$$
\operatorname{AVaR}_{\alpha}\left(\inf _{t \in[0, T]} C_{t}\right) \leq \operatorname{AVaR}_{\alpha}\left(\inf _{t \geq 0} C_{t}\right)=\frac{1}{\alpha} \int_{0}^{\alpha}-\frac{\log \left(\frac{\gamma c}{\lambda} u\right)}{\gamma-\frac{\lambda}{c}} \mathrm{~d} u=\frac{1-\log \left(\frac{\gamma c}{\lambda} \alpha\right)}{\gamma-\frac{\lambda}{c}} .
$$

\subsection{A coherent utility functional induced by an $\mathrm{m}$-stable set}

The concept of m-stability emerges naturally in the study of time-consistency questions for dynamic risk measures. In a discrete-time setup it appears under various names in Artzner et al. [3], Epstein and Schneider [11], Wang [19], Roorda et al. [18], Riedel [16] and Cheridito et al. [6]. In continuous time it is studied in Delbaen [9].

In the following we identify probability measures on $\left(\Omega, \mathcal{F}_{T}, P\right)$ that are absolutely continuous with respect to $P$ with their Radon-Nikodym derivatives $\mathrm{d} Q / \mathrm{d} P$.

Definition 5.1 For $f, g \in\left\{h \in L^{1}\left(\Omega, \mathcal{F}_{T}, P\right) \mid h \geq 0, \mathrm{E}[h]=1\right\}, a[0, T]$-valued stopping time $S$ and $A \in \mathcal{F}_{S}$, we define

$$
f \otimes_{A}^{S} g:=\left\{\begin{array}{ll}
f & \text { on } A^{c} \cup\left\{\mathrm{E}\left[g \mid \mathcal{F}_{S}\right]=0\right\} \\
\mathrm{E}\left[f \mid \mathcal{F}_{S}\right] \frac{g}{\mathrm{E}\left[g \mid \mathcal{F}_{S}\right]} & \text { on } A \cap\left\{\mathrm{E}\left[g \mid \mathcal{F}_{S}\right]>0\right\}
\end{array},\right.
$$

and we call a subset $\mathcal{P}$ of $\left\{h \in L^{1}\left(\Omega, \mathcal{F}_{T}, P\right) \mid h \geq 0, \mathrm{E}[h]=1\right\}$ m-stable if it contains $f \otimes_{A}^{S} g$ for all $f, g \in \mathcal{P}$, every $[0, T]$-valued stopping time $S$ and all $A \in \mathcal{F}_{S}$.

In this example we assume that the filtration $\left(\mathcal{F}_{t}\right)_{t \in[0, T]}$ is generated by a standard Brownian motion $\left(W_{t}\right)_{t \in[0, T]}$ on $(\Omega, \mathcal{F}, P)$, and for every bounded predictable process $q=\left(q_{t}\right)_{t \in[0, T]}$, we denote

$$
\mathcal{E}(q \cdot W)=\exp \left(\int_{0}^{T} q_{u} \mathrm{~d} W_{u}+\frac{1}{2} \int_{0}^{T} q_{u}^{2} \mathrm{~d} u\right) .
$$

It is easy to see that the set

$$
\mathcal{P}_{\sigma}:=\{\mathcal{E}(q \cdot W) \mid q \text { predictable },-1 \leq q \leq 1\}
$$

is m-stable, and it is shown in Delbaen [9] that it is convex and $L^{1}$-closed. For every $[0, T]$-valued stopping time $S$, let $\Theta_{S}$ be the set of all stopping times $\theta$ such that $S \leq \theta \leq T$ and define

$$
\phi_{S}(X):=\operatorname{essinf}_{Q \in \mathcal{P}_{\sigma}, \theta \in \Theta_{S}} \mathrm{E}_{Q}\left[X_{\theta} \mid \mathcal{F}_{S}\right], \quad X \in \mathcal{R}^{\infty} .
$$


Then, $\phi_{0}$ is a coherent utility functional on $\mathcal{R}^{\infty}$, and, as shown in Delbaen [9], it follows from the $\mathrm{m}$-stability of $\mathcal{P}_{\sigma}$ that the following Bellman principle holds true:

$$
\phi_{0}(X)=\phi_{0}\left(X 1_{[0, S)}+\phi_{S}(X) 1_{[S, T]}\right) \quad \text { for all } X \in \mathcal{R}^{\infty} .
$$

In the remainder of this subsection we show that $\phi:=\phi_{0}$ can be extended to a coherent utility functional on $\mathcal{R}^{0}$ and give a simple application. $\phi$ can be written as

$$
\phi(X)=\inf _{a \in \mathcal{Q}_{\sigma}}\langle X, a\rangle
$$

where

$$
\mathcal{Q}_{\sigma}:=\left\{\left(0, E\left[f \mid \mathcal{F}_{\theta}\right] 1_{\{\theta \leq t\}}\right) \mid \theta \in \Theta_{0}, f \in \mathcal{P}_{\sigma}\right\}
$$

Since $\mathcal{F}_{0}$ is trivial, it is obvious that $\mathcal{Q}_{\sigma}$ satisfies condition (2) of Corollary 3.10. Therefore, $\phi_{\text {ext }}$ is a coherent utility functional on $\mathcal{R}^{0}$. By Girsanov's theorem, for all predictable processes $q$ such that $-1 \leq q \leq 1$,

$$
W_{t}^{q}:=W_{t}-\int_{0}^{t} q_{u} \mathrm{~d} u, \quad t \in[0, T]
$$

is a standard Brownian motion under $\mathcal{E}(q \cdot W) \cdot P$. This shows that $\inf _{t \in[0, T]} W_{t}$ is $Q$-integrable for all $Q \in \mathcal{P}_{\sigma}$, from which it follows by Lebesgue's dominated convergence theorem that

$$
\phi_{\text {ext }}(W)=\inf _{Q \in \mathcal{P}_{\sigma}, \theta \in \Theta_{0}} \mathrm{E}_{Q}\left[W_{\theta}\right]
$$

Hence,

$$
\begin{aligned}
\phi_{\text {ext }}(W) & =\inf _{q \text { predictable, }-1 \leq q \leq 1 ; \theta \in \Theta_{0}} \mathrm{E}\left[\mathcal{E}(q \cdot W) W_{\theta}\right] \\
& =\inf _{q \text { predictable, }-1 \leq q \leq 1 ; \theta \in \Theta_{0}} \mathrm{E}\left[\mathcal{E}(q \cdot W)\left(W_{\theta}^{q}+\int_{0}^{\theta} q_{u} \mathrm{~d} u\right)\right] \\
& =-T .
\end{aligned}
$$

\section{References}

1. Artzner, P., Delbaen, F., Eber, J.M., Heath, D.: Thinking coherently. Risk 10, 68-71 (1997)

2. Artzner, P., Delbaen, F., Eber, J.M., Heath, D.: Coherent measures of risk. Math. Financ. 9, 203-228 (1999) 
3. Artzner, P., Delbaen, F., Eber, J.M., Heath, D., Ku, H.: Coherent multiperiod risk adjusted values and Bellman's principle. Preprint, http://www.math.ethz.ch/ delbaen/ (2004) Ann. Oper. Res. (to appear)

4. Asmussen, S.: Ruin Probabilities. Advanced Series on Statistical Science and Applied Probability, vol. 2. World Scientific, Singapore (2000)

5. Cheridito, P., Delbaen, F., Kupper, M.: Coherent and convex risk measures for bounded càdlàg processes. Stoch. Proc. Appl. 112, 1-22 (2004)

6. Cheridito, P., Delbaen, F., Kupper, M.: Dynamic monetary risk measures for bounded discretetime processes. Electron. J. Probab. 11, 57-106 (2006)

7. Delbaen, F.: Coherent risk measures on general probability spaces. In: Advances in Finance and Stochastics, pp. 1-37. Springer, Berlin Heidelberg New York (2002)

8. Delbaen, F.: Coherent Risk Measures. Cattedra Galileiana. Scuola Normale Superiore, Classe di Scienze, Pisa (2000)

9. Delbaen, F.: The structure of $m$-stable sets and in particular of the set of risk neutral measures. In: Séminaire de Probabilités XXXIX, Lecture Notes in Mathematics vol. 1874, pp. 215-258. Springer, Berlin Heidelberg New York (2006)

10. Dellacherie, C., Meyer, P.A.: Probabilities and Potential B, Chap. V-VIII. North-Holland, Amsterdam (1982)

11. Epstein, L.G., Schneider, M.: Recursive multiple-priors. J. Econ. Theory 113, 1-31 (2003)

12. Föllmer, H., Schied, A.: Convex measures of risk and trading constraints. Financ. Stoch. 6, 429-447 (2002)

13. Föllmer, H., Schied, A.: Robust preferences and convex measures of risk. In: Advances in Finance and Stochastics, pp. 39-56. Springer, Berlin Heidelberg New York (2002)

14. Föllmer, H., Schied, A.: Stochastic finance, an introduction in discrete time. Studies in Mathematics, vol. 27. Walter de Gruyter, Berlin (2002)

15. Frittelli, M., Rosazza Gianin, E.: Putting order in risk measures. J. Bank. Financ. 26, 1473-1486 (2002)

16. Riedel, F.: Dynamic coherent risk measures. Stoch. Proc. Appl. 112, 185-200 (2004)

17. Rolski, T., Schmidli, H., Schmidt, V., Teugels, J.: Stochastic Processes for Insurance and Finance. Wiley, Chichester (1999)

18. Roorda, B., Schumacher, J.M., Engwerda, J.: Coherent acceptability measures in multiperiod models. Math. Financ. 15, 589-612 (2005)

19. Wang, T.: Conditional preferences and updating. J. Econ. Theory 108, 286-321 (2003) 